\author{
Miguel Arantes Normanha Filho \\ Mestre em Administração de Serviços - UNIBERO; \\ Mestrando em Gerontologia - PUC-SP; \\ Pós-Graduado em Administração de Marketing - FECAP; \\ Pós-Graduado em Formação de Professores para o Ensino Superior (lato sensu); \\ Coordenador de Tecnologia em Gestão de Marketing. Professor - Uninove. \\ mig.arantes@uninove.br
}

\begin{abstract}
Resumo
Este artigo foca a questão da permanência ou reinserção do idoso, com satisfação e dignidade, no mercado de trabalho. Advoga o uso da experiência adquirida pelo idoso ao longo dos anos como multiplicador de conhecimento, trazendo como conseqüência a permanência das novas gerações nas comunidades e um desenvolvimento sustentável e, de forma secundária, porém relevante, a valorização e a perpetuação da cultura local. $\bigcirc$ artigo foi gerado a partir das abordagens, estudos e ensinamentos adquiridos nas disciplinas: Comunidade e poder local: reconfigurações e resignificações e $O$ estado e o envelhecimento, ministradas no Programa de Mestrado em Gerontologia da Pontifícia Universidade Católica de São Paulo (PUC-SP). Na evolução dos estudos, ficou demonstrada a necessidade de outras investigações, que deverão estar em consonância com o fortalecimento e o universo da comunidade e com a manutenção da cultura local, sem que haja retorno ao passado.
\end{abstract}

Palavras-chave

Comunidades. Cultura local. Idoso. Trabalho.

Normanha FILHo, Miguel Arantes. A permanência ou reinserçãodo idoso no mercado de trabalho: uma alternativa para comunidades voltadas ao desenvolvimento sustentável e à valorização da cultura local. Revista Gerenciais. v. 3, p. 79-86. São Paulo: Uninove, out. 2004. 


\title{
Permanence or reinsertion of the aged in the labor market: an alternative for communities directed to the sustainable development and for valuation of the local culture
}

\begin{abstract}
This study focuses on the permanence, or reinsertion of aged people, with satisfaction and dignity, in the labor market. By the experience acquired through the years acting as a multiplier of their knowledge, bringing, as a consequence, the permanence of the new generations in the communities, causing sustainable development and, in a secondary form, but also relevant, the valuation and perpetuation of the local culture. The study was generated from approaching, studies, and instruction through the following subjects: Community and Local Power: Reconfiguring and a New Signification and The State and the Aging, during the Gerontology course of PUC-SP. The evolution of the study shows the need for more thorough approach to the subject. Those inquiries should be fortifying the community, with the maintenance of the local culture. Without that there would be the sense of return to the past.
\end{abstract}

Key words

Aged people. Communities. Local culture. Work. 


\section{Introdução}

Otual envelhecimento global é um processo sem precedentes, previsível e perfeitamente viável: não é nenhuma crise. Os políticos agem como se essa importante alteração demográfica, com suas enormes implicações econômicas e sociais, pudesse ser colocada à margem nos debates do bem estar social.

(Folha Informativa Terceira Idade E Desenvolvimento, 1999, p. 1).

Em um mercado de trabalho no qual o preconceito relacionado com a idade do idoso é fato concreto, o Brasil, em ritmo crescente, temse destacado pela longevidade de sua população, deixando de ser, gradativamente, um país de jovens. Paralelamente, a persistir esse preconceito, a exclusão do mercado dessa população ainda ativa constituirá um grave problema, de contornos incalculáveis. Com base nas atuais projeções demográficas e estudos sobre o nível de expectativa de vida, percebe-se que tanto o Estado, entendido aqui estritamente como o formulador de diretrizes e ações para os idosos, quanto a sociedade, organizada (terceiro setor, movimentos de base etc.) ou não, e as comunidades de diferentes concepções - associações de moradores de áreas carentes espalhadas pelo Brasil, comunidades de pescadores e artesãos, de igrejas e cidades com vocações históricas para determinadas atividades econômicas - industrialização especializada, agroindustrial e comercial, entre tantas outras -, nas quais podemos fixar uma análise mais centrada e restrita, sem deixar, entretanto, de ser representativa, possuem um mercado "[...] preparado para absorver esse contingente de idosos que deseja e necessita permanecer produtivo [...]" (PAVANI, p. 188, 2002).

\section{A nossa realidade}

Os números brasileiros sobre envelhecimento são alarmantes. Segundo os dados do relatório Sintese de indicadores sociais do Instituto Brasileiro de Geografia e Estatística (IBGE, 2001), em 25 anos a população de idosos poderá ser superior a 30 milhões de pessoas. Apesar de preocupantes, esses dados, com outro olhar e interpretação, poderão quebrar um paradigma hoje alicerçado no preconceito, em particular dos potenciais empregadores, para a não-contratação de profissionais com mais de 60 anos ou até a partir dos 40 anos. É possível tal situação ser revertida?

Sawaia (1999, p. 74-75), na obra As artimanhas da exclusão - análise psicossocial e ética da desigualdade social, chega a qualificar essa situação como verdadeira desclassificação social:

Como a desclassificação social é uma experiência humilhante, ela desestabiliza as relações no seio da comunidade familiar que podem ser afetadas, pois é difícil para alguns admitir que não estejam à altura das pessoas que o cercam [...] a fragilidade pode levar a uma fase de dependência, já que a precariedade profissional, particularmente quando é durável, acarreta uma diminuição de renda e uma degradação das condições de vida que pode em parte ser compensada pelos serviços sociais.

Que serviços sociais são esses, em um Brasil desigual com péssima e precária distribuição de renda, sem mencionar a pré-falimentar assistência social?

Por mais que não desejemos admitir, a relação de nossa sociedade com o idoso,

[...] com sua cultura de exclusão, deixa à parte esse outro que ninguém quer como espelho porque, talvez, anuncie a possibilidade do próprio futuro que cada um pode ter [...] (MONTEIRO, 2001, p. 31-32). 
Muitas vezes, não nos atemos aos significados de palavras e termos, entre eles 'técnica', definido pelo Dicionário Houaiss da língua portuguesa (2003, p. 502) como "[...] habilidade especial para se executar algo." Quantos idosos com capacidade produtiva possuem técnicas não formalizadas e documentadas que podem ser transmitidas de geração para geração, com efeito multiplicador, sem perda ou adequação de características diferenciadoras e sofisticadas para o contexto produtivo de certas comunidades? Em momento algum, tal situação se contrapõe ao observado por Kliksberg (2002, p. 23-24):

A humanidade chega ao final do século $\mathrm{XX}$ com progressos de enorme grandeza e profundidade em suas capacidades científicas, tecnológicas e produtivas. Produzem-se rupturas epistemológicas simultâneas em numerosos campos do conhecimento, que estão gerando modelos conceituais renovados para se compreender os fenômenos, e uma nova onda de tecnologias, baseadas em conhecimento de amplíssimas possibilidades [...] Contudo, o imenso potencial de capacidade produtiva não está se transformando em melhoria nas difíceis condições de vida de amplos setores do planeta. Existe uma brecha enorme entre esse potencial e a vida cotidiana.

A reflexão que nos conduz obriga à análise de qual técnica é relevante para uma potente capacidade produtiva e rentável. Assim, o contexto de cada comunidade, o local, é fator determinante da relevância, significado e ressignificado de técnica para dada ação produtiva, e não exclusivamente uma sofisticada tecnologia para um país de primeiro mundo.

\section{Globalização e comunidade}

Em busca de uma melhor clarificação de análise, é importante observar que nem tudo deve, de forma leviana, ser creditado ao efeito da globalização econômica (entendida aqui por neoliberalismo). Não que seus efeitos não tenham sido e não são devastadores para as economias, em especial às dos países do Terceiro Mundo como - Brasil; ao contrário, ela possui intencionalidade e é ideológica. No entanto, ao buscar caminhos, devemos descentralizar o debate sobre a globalização econômica e centrar nossa atenção na dimensão social, política e cultural; assim, teremos uma nova visão com alternativas múltiplas para a sociedade com todas as várias situações que se apresentam, se criam e se multiplicam.

$\mathrm{Na} 4^{\mathrm{a}}$ edição da obra Território, globalização e fragmentação, numa abordagem que resgata com bastante clareza o espaço físico do território, da resistência da sociedade civil e da comunidade, Milton Santos (2000, p. 16-19) diz que:

\begin{abstract}
Os territórios são formas, mas os territórios usados são objetos e ações, sinônimo de espaço humano, espaço habitado [...] encontramos no território, hoje, novos recortes, além da categoria região [...] As horizontalidades serão os domínios da contigüidade e verticalidades. As horizontalidades serão os domínios da contigüidade, daqueles lugares, vizinhos reunidos por uma continuidade territorial [...] Por enquanto, o lugar - não importa sua dimensão - é [...] resistência da sociedade civil [...] Para isso, é indispensável insistir na necessidade de conhecimento sistemático da realidade, mediante o tratamento analítico desse seu aspecto fundamental que é o território (o território usado, o uso do território).
\end{abstract}

Segundo Boaventura de Sousa Santos (2002, p. 63), na obra Globalização e as Ciências Sociais, 
[...] o global acontece localmente, e que não existe condição global para qual não consigamos encontrar uma raiz local [...] uma inserção cultural especifica [...]

Temos, portanto, pela análise de Boaventura, um novo olhar sobre a comunidade e a cultura local, preservada, ativa e ao mesmo tempo inserida no global, por meio de atividades econômicas.

Nessa medida, não é utópico pensar em comunidades voltadas para o desenvolvimento sustentável e para a valorização da cultura local, em que o idoso se torna elemento fundamental de inserção e ressignificação do trabalho, da globalização, da cultura e da dignidade e, ao mesmo tempo, da satisfação pessoal e lazer, na direção de sentir-se bem com tudo aquilo que faz, melhorando a qualidade social de vida. Para que tal fato ocorra, a comunidade deve ter outro significado, conforme a formulação feita por Silva (2003), em sala de aula, após discussão sobre o tema comunidade. A autora, por meio de uma perspectiva histórica, propõe estudar a retomada atual do conceito de comunidade, no limiar do século XXI, nos quadros contraditórios da globalização,

Comunidade na perspectiva de articulação local global e de dimensão temporal/histórica entendida como um espaço de convivência, ou seja, de processos vínculos e relações sociais heterogêneas, contraditórias, conflitivas e de cooperação, onde no cotidiano da vida interpessoal e intergrupal, de terem valores, sentimentos, redes de interesses e poderes diversos, que organizados e mobilizados, constroem/reconstroem subjetividade e intersubjetividades que levam ações coletivas participadas na concretização de objetos imediatos e mediados comuns para a melhoria da qualidade de vida a todos, e na concretização de uma sociedade democrática radical (justa e solidária).

\section{Contribuição do trabalhador idoso: uma possibilidade}

Prospectando cenários, podemos fazer a seguinte proposição: o trabalhador idoso é capaz de contribuir para o desenvolvimento sustentável de comunidades e para a valorização da cultura local.

Essa proposição pressupõe que 0 trabalhador idoso tem seu conhecimento construído e valorizado por um somatório de elementos, entre eles o período de tempo no exercício profissional, a educação formal e autodidata a que teve acesso, a cultura e o local que impregnaram sua vida ao longo de sua vivência na comunidade em que mora e trabalha, perpetuando, por sua ação multiplicadora, o conhecimento adquirido. Não precisarão migrar das comunidades, nas quais nasceram e cresceram, para os grandes centros urbanos, onde os conflitos sociais transcorrem com muita evidência.

Os produtos quase artesanais elaborados por micro e pequenas empresas possuem alto valor agregado quando comercializados e distribuídos com o auxílio positivo da globalização, do avanço tecnológico e de organizações do terceiro setor e da organização comunitária em rede. As empresas, no contexto da proposição formulada, podem ser classificadas

[...] segundo as suas dimensões em micro, pequenas, médias e grandes, porém uma 'Pequena e Microempresa' (Peme) pode pertencer tanto a um empresário muito capaz, e desse ponto de vista, ser um grande empresário, quanto a um empresário imaturo em alguma área [...] (Montaño, 2001).

Além disso, podem compor-se de trabalhadores experientes, idosos capazes de ensinar técnicas produtivas e serviços às novas gerações.

Não é impossível pensar em tal significado, na eliminação da pobreza ética social, conforme expõe Buarque (2000, p. 59): 
Toda a teoria econômica aprendida pelos jovens doutores latino-americanos, em países onde o problema da pobreza foi basicamente resultado do tema do aumento de riqueza, ignorando completamente o caminho para uma redução da pobreza. Concentrada na riqueza, a teoria econômica sofre uma crônica pobreza ética. É incapaz de entender e enfrentar o problema da pobreza social.

Este estudo não possui a finalidade de questionar a Consolidação das Leis do Trabalho (CLT), ou a Justiça do Trabalho nem os encargos diretos e indiretos que incidem na remuneração do trabalhador formal, proporcionando uma outra discussão sobre o chamado 'custo-Brasil', tampouco os efeitos perversos do capitalismo que, historicamente, nunca foram tão selvagens. Trata-se, isto sim, de lançar um novo olhar, um novo paradigma não assistencialista, mas concreto e viável no que se refere à extensão do uso, com produtividade contextualizada, dignidade, lazer, poder (local) e manutenção da cultura comunitária, via acúmulo de conhecimento e de técnicas do trabalhador idoso, sem que represente, entretanto, a perda de espaço para os jovens a ser incorporados ao mercado de trabalho. E tal análise se sustenta no exposto por Oliven (2002, p. 26) sobre a realidade brasileira no que se refere às limitações de sua política econômica como indutora na geração de emprego formal:

Dada entretanto a incapacidade da economia de países como o Brasil de oferecer empregos regulares a sua população urbana com idade de trabalhar, existe uma parte considerável da força de trabalho que sobrevive no chamado setor informal de trabalho [...] o trabalho 'por conta própria' [grifo nosso] e de não implicar em disciplina e autoridade de trabalho, de servir pessoas que às vezes teriam dificuldade de obter empregos no setor formal de trabalho (mulheres, crianças, idosos, deficientes físicos etc) e de permitir ter várias atividades simultaneamente (inclusive a de trabalhar no setor formal e no informal).

Uma nova visão do trabalho, do papel produtivo do idoso, da cultura e do poder local proporcionará uma nova leitura da crise do sistema capitalista, conforme aborda Reale (2000, p. 15):

[...] a superação da crise do capitalista depende tanto de providências econômicas quanto de determinações éticas e políticas, a fim de que não se assista ao drama de um mundo no qual cada novo progresso tecnológico imposta em redução nos postos de trabalho, com fria e inexorável conseqüência da redução do número de máquinas indispensáveis à produção. É possível que tais desequilíbrios possam vir a ser superados graças apenas às leis competitivas do mercado, mas as necessidades vitais dos trabalhadores e de suas famílias que não podem aguardar indefinidamente os reajustes espontâneos pregados pelos mentores do neoliberalismo.

Visão do trabalho, com foco na comunidade e no poder local, como descrito por Silva (2003), conduz também, e obrigatoriamente, ao repensar da comunidade e de seus atores, com a reinserção do idoso no mercado de trabalho. Tudo faz parte de uma grande equação social e econômica, cujas variáveis não funcionarão em separado. Wanderley (1998, p. 127) afirma que:

princípio comunitário atravessa as sociedades e os tempos. À medida que forem surgindo as 'sociedades de massa' [grifo nosso] que se formaram as concentrações populacionais em virtude da industrialização e da urbanização, que aconteceu à Primeira Guerra Mundial, várias correntes de idéias lançaram suspeitas sobre o mundo da ciência e da técnica, o declínio do Ocidente, o 
racional que estaria eliminando o vital e o efetivo. Ora, nada melhor então que a comunidade para salvaguardar a amizade, a intimidade, a partilha da vida, as relações pessoais. A família surge com o modelo de vida comunitária, mas a idéia de comunidade se estende à vizinhança, ao bairro, ao grupo social, chegando-se a transpô-la para âmbitos mais abrangentes - comunidade nacional e internacional.

Haverá sempre vários tipos de trabalhadores idosos, com competências diferentes, todos inseridos no mercado, com suas múltiplas facetas e características. A proposição do presente estudo pode ser uma das muitas formas que estudiosos poderão refutar, aprimorar e de outras formulações a serem propostas para a exclusão social e desigualdade, que estão presentes nos nossos dias, podendo ser retratadas nos termos de Pochmann e Amorin (2003, p. 21),

Quando esparramado pelo mapa o conjunto de indicadores, chega-se a uma singela conclusão a respeito do caleidoscópio que representa a exclusão social no Brasil. Na melhor tradição de Josué de Castro e Milton Santos, que procuraram destacar a recorrente manifestação da desigualdade, verifica-se que, em pleno limiar do terceiro milênio, o Brasil continua a se equilibrar sobre uma frágil base como nação.

\section{Considerações finais}

Para além de meras reflexões sobre o problema, o objetivo deste estudo foi apresentar possíveis soluções, mesmo que incipientes e sem uma rigorosa metodologia científica.

Não podemos mais manter o olhar na velhice como fato consumado de exclusão, por fator biológico ou por fator precoce, por conta de um mercado de trabalho preconceituoso e 'míope', que fixa patamares de 40 ou 50 anos para o fim da etapa produtiva do trabalhador. É necessário também abandonar a visão puramente assistencialista dos projetos e programas voltados a essas faixas etárias, pois elas, apesar de necessárias em algumas situações - instituições asilares, ações médico-geriátricas, aposentadorias, por exemplo -, em vez de proporcionarem um melhor padrão de vida a quem sempre trabalhou, resultam em perda da sociabilidade, quando não em 'morte social' motivada por uma sociedade totalmente alheia e despreparada para a questão.

$\mathrm{Na}$ evolução do estudo, ficou demonstrada a necessidade de outras investigações profundas sobre tópicos que abordem, entre outros:

- Como é hoje a ocupação do idoso no mercado de trabalho?

- Como é o fator de exclusão do idoso por 'idade biológica' x 'idade precoce'?

- Qual é o novo paradigma ou novo olhar necessário para a manutenção ou inserção do trabalhador idoso no mercado de trabalho?

- Qual é o limite entre trabalho e lazer para o idoso em uma comunidade objeto da proposição?

- Qual é o papel do Terceiro Setor na questão?

- Qual é o novo conceito de trabalho e ocupação para o idoso na questão do saber (capital intelectual), no trabalho autônomo, como microempresário, no trabalho em cooperativas, ou em outras formas?

Tais estudos deverão estar em consonância com o fortalecimento e o universo da comunidade e com a manutenção da cultura local, sem que haja o sentido de retorno ao passado, e sim uma nova forma de comunidade, talvez híbrida, mas sem dúvida com um foco novo que contemple novas necessidades. 


\section{Referências}

BuArque, Cristovam. El camino provisional. In: OdIo, Rodrigo Carazo (Org.). La tercera via. Cartago: LUR, 2000 .

Folha Informativa TERCEIRA IDADE E DESENVOLVIMENTO. Terceira idade e desenvolvimento: envelhecimento global sem precedentes. p. 1. London: s/n., 1999.

HouAlss, Antônio. Dicionário Houaiss da língua portuguesa: míni. Rio de Janeiro: Objetiva, 2003.

InSTITUto Brasileiro dE Geografia E Estatística (IBGE). Síntese de indicadores sociais. Rio de Janeiro: s/n., 2001.

KLIKSBERG, Bernardo. Repensando o estado para o desenvolvimento social: superando dogmas e convencionalismos. 2. ed. São Paulo: Cortez, 2002.

Montaño, Carlos. Microempresa na era da globalização. 2. ed. São Paulo: Cortez, 2001.

Monteiro, Pedro Paulo. Envelhecer: histórias, encontros, transformações. Belo Horizonte: Autêntica, 2001.

Oliven, Ruben George. A antropologia de grupos urbanos. 5. ed. Petrópolis: Vozes, 2002.
Pavani, Regina M. Girelli. A imagem da velhice. In: Trevisan, Leonardo; CAstro, Maria da Conceição de Araújo (Org.). Transformações no trabalho. São Paulo: Olho d'Água, 2002.

Pochmann, Marcio; Amorin, Ricardo (Org.). Atlas da exclusão social no Brasil. São Paulo: Cortez, 2003.

Reale, Miguel. Crise do capitalismo e crise do Estado. São Paulo: SenAC, 2000.

SANTOS, Boaventura de Sousa. Globalização e as ciências sociais. São Paulo: Cortez, 2002.

SAntos, Milton. Território, globalização e fragmentação. São Paulo: Annablume, 2000.

SaWAIA, Bader (Org.). As artimanhas da exclusão: análise psicossocial e ética da desigualdade social. 3. ed. Petrópolis: Vozes, 1999.

Silva, M. Lúcia da. Conceito de comunidade. Anotações de aula: comunidade e poder local - reconfigurações e resignificações. São Paulo: PUC-SP, 30 maio 2003.

Wanderley, Mariângela Belfiore. Metamorfoses do desenvolvimento de comunidade. 2. ed. São Paulo: Cortez, 1998. 